\title{
TOPOLOGIA DOS ORBITAIS MOLECULARES DE POLIENOS PARA DEDUZIR ENERGIAS RELATIVAS E AVALIAR AROMATICIDADE
}

\author{
Silvio Cunha*,\# \\ Instituto de Química, Universidade Federal da Bahia, Campus de Ondina, 40170-115 Salvador - BA, Brasil
}

Recebido em 15/02/2018; aceito em 21/03/2018; publicado na web em 10/04/2018

\begin{abstract}
TOPOLOGY OF MOLECULAR ORBITAL FOR DEDUCING RELATIVE ENERGIES FROM POLYENE AND EVALUATING AROMATICITY. A didactic alternative approach is proposed for deducing the molecular orbital topology of acyclic and cyclic polyenes and then evaluating their bonding, antibonding, and nonbonding character. The relative energies of molecular orbitals of neutral molecules and ions were described, in addition to the orbital degeneracy to cyclic conjugated polyenes, and the method deduce correctly the relative stability of polyenes. This alternative method complements the lack of explanation of a substantial number of organic chemist undergraduate textbooks in relation to orbital degeneracy in benzene and is a pedagogical approach to teach aromaticity and antiaromaticity.
\end{abstract}

Keywords: aromaticity; undergraduate organic chemistry teach; orbital topology.

\section{INTRODUÇÃO}

Na prática docente vivenciamos situações recorrentes ao longo da carreira onde estudantes, temporal e geograficamente distintos, apresentam as mesmas dificuldades de compreensão de conceitos e fenômenos relacionados à química orgânica. ${ }^{1}$ Antes de ser uma limitação intrínseca dos estudantes, a origem destas dificuldades pode residir na forma como esses conceitos e fenômenos são apresentados aos graduandos, ${ }^{2}$ bem como da sofisticada representação pictórica da fenomenologia química. ${ }^{3}$

A aromaticidade e a degenerescência dos orbitais moleculares do benzeno e correlatos se enquadram neste cenário pois, nos textos didáticos, são tão somente apresentados como fatos, e o fato passa a ser a explicação sem uma dedução que possa levar à compreensão. Isto é particularmente deletério, pois a química dos compostos aromáticos e o fenômeno da aromaticidade são temas balizares na formação dos profissionais da química, ${ }^{4}$ ensinados nos cursos teóricos de química orgânica, que deve resultar na compreensão da estabilidade e reatividade diferenciada do anel benzênico, quando comparado aos alcenos. 5

Em continuação à nossa abordagem de ensino de orbitais moleculares nos cursos básicos de química orgânica na graduação, ${ }^{6-8}$ apresentamos aqui um método operacional para avaliar as energias relativas dos orbitais moleculares de hidrocarbonetos aromáticas e antiaromáticas, e dos alcenos conjugados correspondentes, Figura 1.

A proposta se baseia na construção da topologia dos orbitais moleculares ${ }^{9}$ que representa a combinação linear dos orbitais atômicos p do carbono, permitindo deduzir a natureza ligante e antiligante de cada orbital e avaliar qualitativamente suas energias relativas e, assim, comparar a energia total de estabilização dos elétrons dos polienos cíclicos conjugados e íons orgânicos com a dos correspondes polienos acíclicos. Dessa forma, a degenerescência dos orbitais do benzeno e também a energia relativa dos orbitais dos polienos são deduzidas pelo próprio estudante, suplantando a descrição estática dos livros textos amplamente empregados na graduação. ${ }^{10}$

*e-mail: silviodc@ufba.br

"Programa de Pós-Graduação em Ciências, Energia e Ambiente, Centro Interdisciplinar em Energia e Ambiente, Universidade Federal da Bahia, Campus de Ondina, 40170-115 Salvador - BA, Brasil

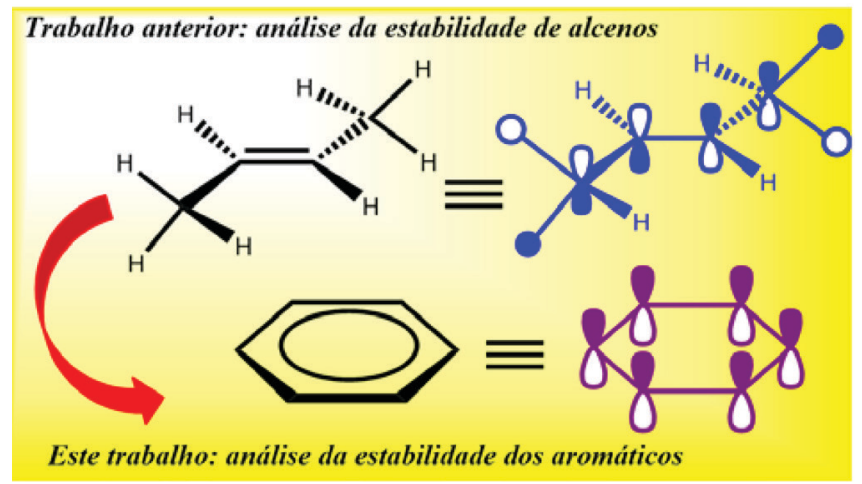

Figura 1. Dos alcenos aos polienos e aromáticos: estabilidade e orbitais moleculares

Critérios apresentados nos livros de graduação para avaliar aromaticidade e energias relativas dos orbitais moleculares

Para verificar o tipo de argumentação didática presente nos textos de graduação, uma amostra representativa de treze livros foi analisada, desde clássicos como o Morrison e Boyd até livros mais recentes, e assim avaliar a perenidade dos critérios empregados na explicação da aromaticidade tendo o benzeno como modelo. ${ }^{10} \mathrm{~A}$ maioria tem tradução para a língua portuguesa, sendo dois de autores brasileiros, e na Figura 2 estão reunidos os recursos gráficos empregados nestes livros para ensinar os argumentos que conduzem o estudante a compreender a maior estabilidade do benzeno, em função da aromaticidade, quando comparada aos polienos de cadeia aberta. Não foi incluída a comparação entre reação de adição a alcenos versos substituição eletrofílica aromática, que evidencia a diferença de reatividade, pois esta não é a explicação da aromaticidade, é a sua consequência.

O critério da estabilidade termodinâmica introduzida por Pauling, ${ }^{11}$ que faz a comparação do calor de hidrogenação, prevalece em todos os livros. Ao empregar este critério para demonstrar a maior estabilidade do sistema de elétrons $\pi$ do anel aromático, comparam o calor de hidrogenação do ciclo-hexeno e do hipotético ciclo-hexatrieno com o do benzeno, e a diferença destas energias é denominada energia de estabilização por ressonância, Figura 2. Em seguida, os textos apresentam o diagrama de energia indicando a existências de 
orbitais moleculares degenerados com a corresponde topologia (em alguns casos é reapresentado sem a topologia). Por fim, concluem que os elétrons $\pi$ no sistema aromático são mais estabilizados no seu conjunto, com menor energia eletrônica que os elétrons $\pi$ dos alcenos conjugados de igual número de átomos de carbono.

O método mnemônico do círculo e polígono desenvolvido por Frost e Musilin ${ }^{12}$ é apresentado na minoria dos textos de graduação, sendo este recurso o único empregado para julgar a degenerescência dos orbitais. Em nenhum deles há uma dedução passo-a-passo para se chegar à conclusão da existência da degenerescência dos orbitais moleculares dos sistemas com elétrons $\pi$ conjugados quando se passa de uma substância acíclica para uma cíclica, ou qualquer discussão sobre a topologia dos orbitais moleculares que conduza e este fim. Adicionalmente, a topologia dos orbitais $\pi$ do benzeno é apresentada sem qualquer explicação de como é construída ou deduzida. Nem mesmo há indicação de que são topologias calculados pelo método de Hückel $^{5,13}$ ou outro método químico-quântico e que representam a combinação linear dos orbitais atômicos p do carbono. A regra de Hucke ${ }^{14}$ é enunciada em todos os livros analisados, mas a consequência do "número mágico" de elétrons $\pi$ do sistema conjugado monocíclico, que resulta no comportamento aromático, é representada graficamente de formas diversas nestes mesmos livros.

Ao aplicar todas estas considerações os livros procuram contemplar os critérios energéticos e estruturais necessários à compreensão da aromaticidade. Apesar desta estratégia de apresentação e conceituação ser largamente empregada, os textos de graduação não demonstram como avaliar a energia de estabilização dos elétrons $\pi$ nos polienos cíclicos que seguem a regra de Hückel, quando comparados aos respectivos alcenos acíclicos de igual número de átomos de carbono e de elétrons $\pi$ conjugados. Por outro lado, nos cursos avançados de química orgânica na pós-graduação e mesmo na graduação, é possível lançar mão de expressões matemáticos e/ou de pacotes computacionais baseados nos métodos químico-quânticos para calcular as energias dos orbitais moleculares. ${ }^{15-17}$ Entretanto, esta abordagem não é diretamente transponível aos cursos básicos de química orgânica, o que leva à apresentação dos níveis de energia do benzeno, por exemplo, sem qualquer dedução, mesmo que qualitativa.

Do exposto, há, portanto, uma clara lacuna didática nos textos de química orgânica na graduação na explicação das energias dos polienos acíclicos e cíclicos, da degenerescência dos orbitais moleculares e da aromaticidade. Deduzir a topologia dos orbitais moleculares é necessário para, como consequência, deduzir as energias relativas do conjunto dos orbitais em análise. Um método que proporcione o aprendizado de um conjunto de passos que conduza a estas deduções pode ser útil ao estudante. Um procedimento que o dota desta capacidade de análise é a seguir descrito.

Estratégia alternativa para deduzir as energias relativas dos orbitais de polienos e íons cíclicos e acíclicos com elétrons $\pi$ conjugados

A estratégia alternativa para deduzir as energias relativas dos

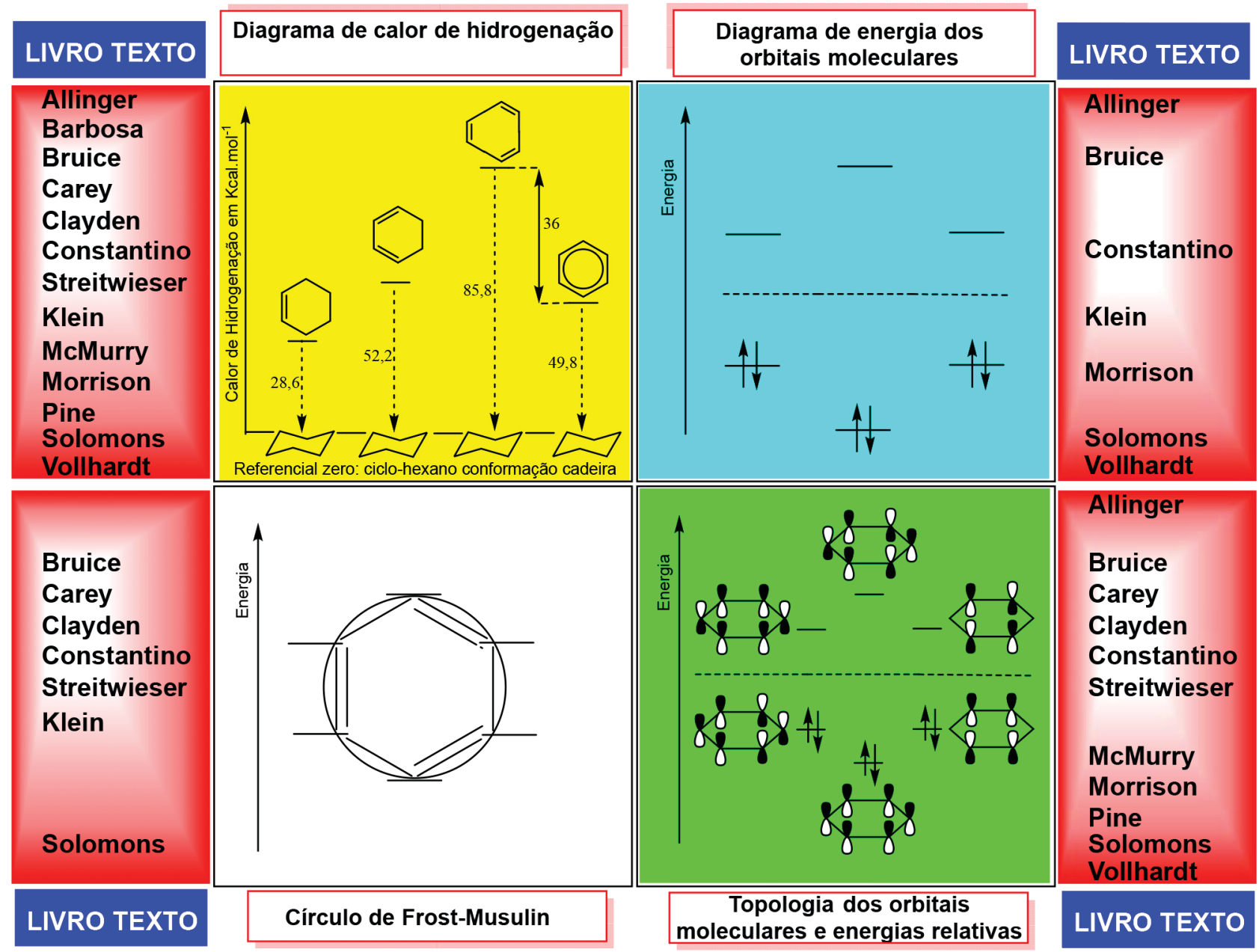

Figura 2. Recursos gráficos empregados nos livros textos de Química Orgânica para explicar a aromaticidade e a degenerescência dos orbitais moleculares do benzeno 
orbitais de moléculas neutras ou espécies iônicas consiste na aplicação dos Passos 1-7 que proporciona deduzir as energias relativas dos orbitais moleculares da maioria dos exemplos tradicionalmente estudados nos textos didáticos da graduação. Para exemplificar, os Passos 1-7 foram aplicados na dedução das energias relativas dos orbitais $\pi$ de moléculas e íons acíclicos conjugados de dois a seis átomos, e em moléculas e íons cíclicos de três a sete membros.

Na execução dos Passos 1-7 as premissas detalhadas na explicação de cada um deles são conhecidas pelos estudantes desde os cursos básicos de química geral onde tópicos de teoria atômica e de ligação química são trabalhados, como a conservação do número total de orbitais atômicos que se combinam para formar os moleculares, a existência de planos nodais nos orbitais moleculares, o cancelamento de uma interação ligante por uma antiligante, e a noção de que quanto maior o número de interações ligantes mais estável deve ser o orbital.

O recomendável é que o estudante inicie a análise com os polienos acíclicos, se aproprie do procedimento descrito nos Passo 1-3 da Figura 3 e então aplique-os ao polieno imediatamente superior em número de átomos, fazendo toda a análise até o sistema com seis átomos, um de cada vez. Para exemplificar a construção do diagrama dos orbitais $\pi$ e dedução das energias relativas, fazemos o detalhamento para o 1,3-butadieno e o cátion ciclopropenila na Figura 3.

O Passo 1 é determinar o número de orbitais atômicos p paralelos e adjacentes da estrutura em análise que dão origem aos orbitais moleculares $\Psi$. Para os sistemas $\pi$ conjugados aqui descritos, cada átomo contribui com um orbital atômico $\mathrm{p}$ para formar o circuito de elétrons $\pi$. Assim, a construção da combinação linear dos orbitais atômicos de um dado sistema de $\mathbf{n}$ átomos na estrutura resulta em $\mathbf{n}$ orbitais moleculares, ou seja, $\Psi_{1}, \Psi_{2}$, até $\Psi_{n}$ (ver Passo 1 da Figura 3 para o anel de três carbonos).

O Passo 2 é desenhar uma estrutura de linhas para cada $\Psi_{n}$.

O Passo 3 consiste em localizar o plano nodal em cada estrutura desenhada, com a máxima simetria possível e, para os polienos acíclicos, são empregados planos verticais que são distribuídos tendo como referencial o centro geométrico do sistema $\pi$ conjugado. Havendo mais de um plano, estes são localizados equidistantes a este centro guardando a máxima simetria possível, como se em cada orbital molecular $\Psi_{\mathrm{n}}$ o centro geométrico do sistema $\pi$ conjugado fosse uma espécie de espelho interno, que reflete cada plano de uma metade do sistema $\pi$ da molécula na outra metade, sendo indicado na Figura 3 para o butadieno. Os planos nodais verticais são distribuídos em número crescente, iniciando em $\Psi_{2}$ pois todo $\Psi_{1}$ não possui plano nodal.

Para os demais polienos acíclicos são empregados planos verticais tantos quantos forem necessários, sempre distribuídos tendo como referencial o centro geométrico do sistema $\pi$ conjugado, sempre localizados equidistantes a este centro, com a máxima simetria possível, como indicado no Passo 3, o que resulta na Figura 4. É por este critério que, quando o sistema $\pi$ conjugado está contido em número ímpar de átomos, alguns planos ficam localizados diretamente no átomo central do sistema.

Aplicando a estratégia descrita para os Passos 1-3 aos polienos conjugados acíclicos, cujo número de planos nodais de um dado orbital molecular $\Psi_{n}$ é n-1, é possível localizar os planos nodais para os entes químicos de dois a seis átomos (o eteno foi incluído na análise como molécula de referência), Figura 4 . O resultado final apresentado na Figura 4 deve ser reproduzido antes que o estudante avance na dedução das energias de cada orbital, para que possa seguir com segurança na etapa da comparação das energias do sistema acíclico com o cíclico abaixo descrita.

No Passo 4 é inferida a topologia dos orbitais moleculares pois, uma vez distribuídos os planos nodais em cada orbital molecular $\Psi_{n}$, os orbitais atômicos p são desenhados em cada átomo de todas as estruturas onde não passe plano nodal, levando-se em conta a existência de inversão do sinal da fase determinada pela presença do plano nodal entre dois carbonos ou passando diretamente por um dos átomos da estrutura, como ilustrado na Figura 3.

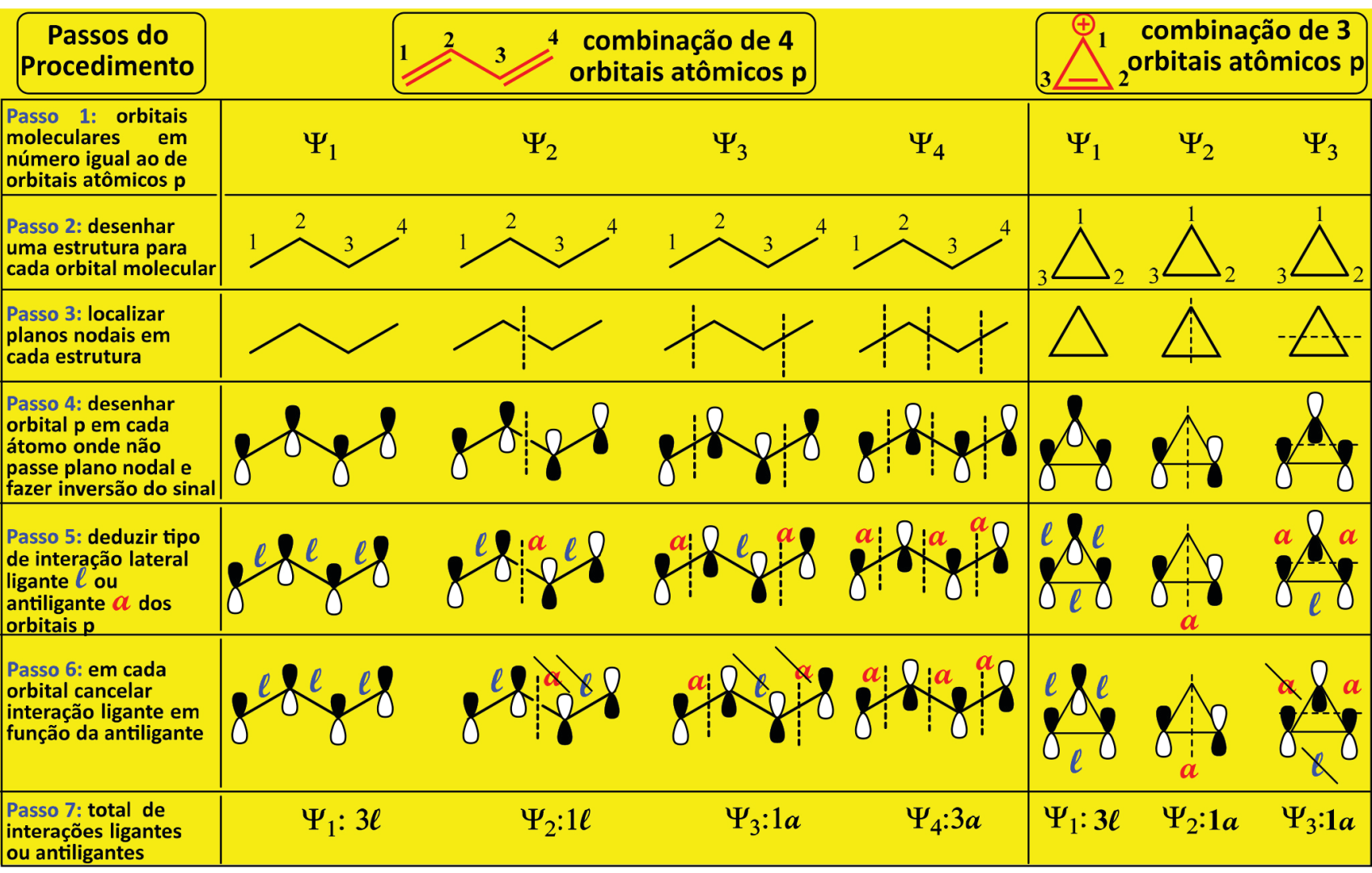

Figura 3. Procedimento alternativo de dedução da topologia dos orbitais $\pi$ e das energias relativas para o 1,3-butadieno e cátion ciclopropenila 


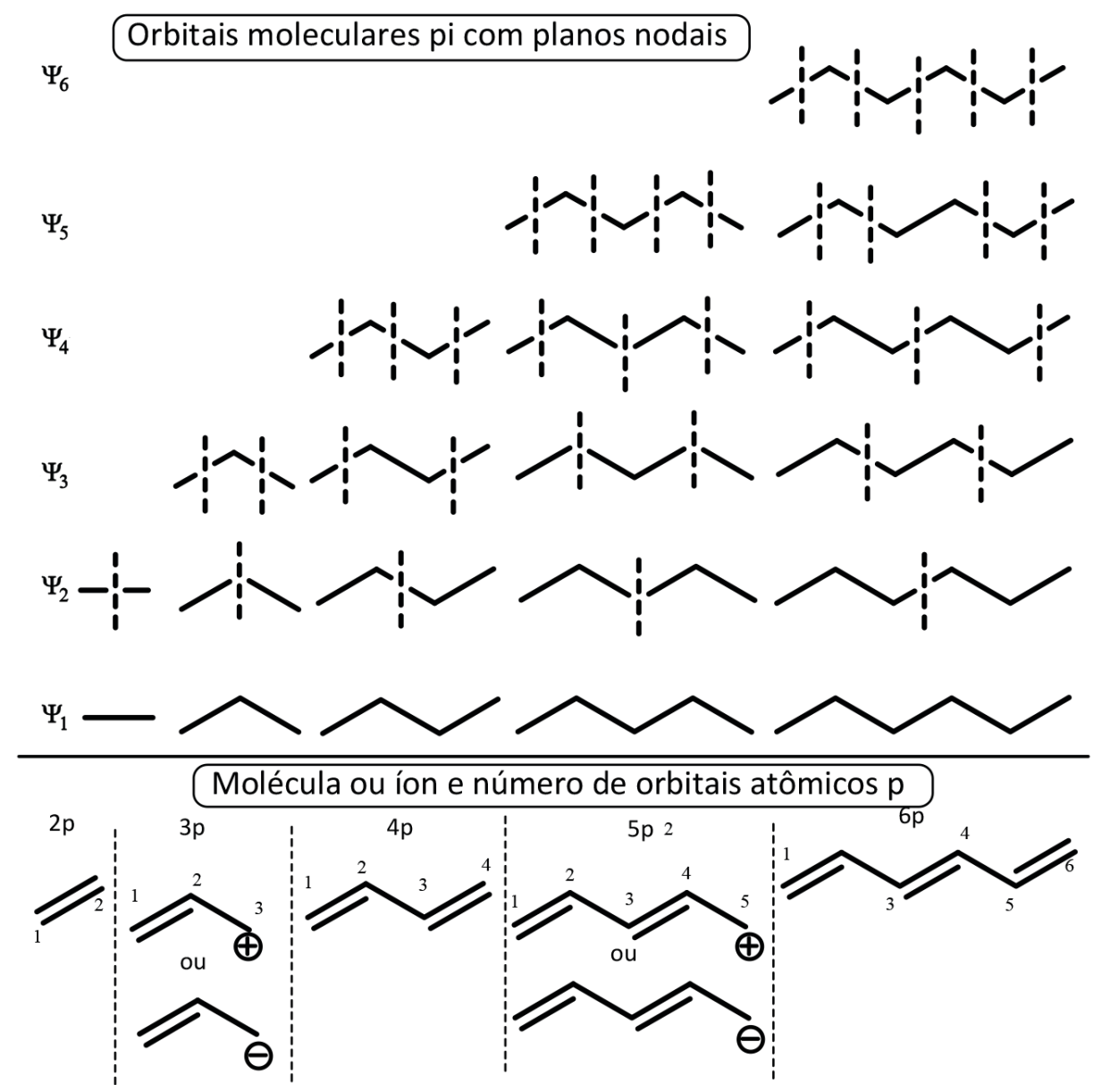

Figura 4. Localização dos planos nodais dos orbitais $\pi$ para moléculas e íons acíclicos de 2 a 6 átomos empregando os Passos 1-3 do procedimento alternativo

No Passo 5, cada interação adjacente construtiva (em fase) dos orbitais atômicos p resulta em uma interação ligante energeticamente favorável à estabilização do sistema, designada aqui pela letra $\boldsymbol{\ell}$. No caso contrário, quando a interação adjacente ocorre com os orbitais atômicos p fora de fase, resulta em uma interação antiligante, energeticamente desfavorável à estabilização do sistema, designada aqui pela letra $\boldsymbol{a}$.

No Passo 6 são avaliados os orbitais $\Psi_{n}$ que possuem simultaneamente interações ligantes e antiligantes, de forma que uma interação $\ell$ é cancelada por uma $\boldsymbol{a}$.

No Passo 7 é determinado o cômputo do número de interações resultantes em cada orbital molecular $\Psi_{n}$ totalizando as interações ligantes e antiligantes de cada orbital, Figura 3. Quão maior o número de interações ligantes resultante, mais estável é o orbital e menor a sua energia. Quão maior o número de interações antiligantes resultante, maior a energia do orbital. A energia de cada orbital molecular é então expressa em termos de energia ligante $\boldsymbol{\ell}$ e energia antiligante $\boldsymbol{a}$.

Na Figura 5 encontra-se representado o resultado da aplicação dos Passos 1-7 para a série dos compostos acíclicos de dois a seis átomos de carbonos. Para a dedução do valor da energia de um dado orbital é necessário que a designação da interação como ligantes $\ell$ ou antiligantes $\boldsymbol{a}$ atenda rigorosamente o que preconiza o Passo 5. Por exemplo, nos orbital $\Psi_{2}$ do cátion alílico não há interações adjacentes, uma vez que o plano nodal secciona a cadeia no átomo central, o que resulta em densidade eletrônica nula neste carbono neste orbital. Assim, como não existem interações adjacentes entre dois orbitais atômicos, esta combinação gera um orbital molecular cuja energia é a mesma do orbital atômico p, Figura 5. Outra possibilidade de um orbital molecular ter este valor de energia é quando o número de interações $\boldsymbol{\ell}$ é igual a de $\boldsymbol{a}$.
Para fins de comparação, a estratégia alternativa foi estendida a moléculas e íons cíclicos, e assim poder comparar a energia total de estabilização dos elétrons dos polienos cíclicos conjugados com os correspondes polienos acíclicos. A execução dos Passos 1 e 2 é idêntica para os sistemas acíclicos e cíclicos, mas para o Passo 3 é preciso uma pequena adaptação para os polienos cíclicos, pois são empregados planos verticais e horizontais, distribuídos nesta ordem, de forma alternada e em número crescente, iniciando em $\Psi_{2}$. Assim, para a estrutura que corresponde a $\Psi_{2}$, localizar o plano vertical que divide a estrutura ao meio na vertical; em seguida, para $\Psi_{3}$ localizar o plano horizontal que divide a estrutura ao meio na horizontal. Ambos os planos passam pelo centro geométrico da estrutura, como ilustrado na Figura 3 (ver Passos 1 a 3 para o anel de três carbonos).

Caso a estrutura admita um maior número de orbitais moleculares $\Psi_{n}$, empregar dois planos nodais na vertical para $\Psi_{4}$ seguidos de dois na horizontal para $\Psi_{5}$, que são localizados equidistantes do centro geométrico da estrutura e o mais afastado possível deste centro, tanto na vertical quanto na horizontal. Quando for necessário empregar três planos nodais, o processo passa a ser, para o novo orbital $\Psi_{6}$, uma combinação das localizações dos planos verticais de $\Psi_{2}$ com $\Psi_{4}$ seguido dos planos horizontais de $\Psi_{3}$ com $\Psi_{5}$ para o novo $\Psi_{7}$. Na Figura 6 estão descritos os Passos 1-3 para os monociclos de três a sete átomos de carbonos.

É importante destacar que as estruturas devem ser escritas com um dos lados para baixo, exceto para o anel de quatro átomos, que é escrito com um vértice para baixo, Figura 4, e que a localização dos planos sempre inicia em $\Psi_{2}$ pela vertical, mantendo-se daí em diante a alternância vertical/horizontal até preencher todos orbitais moleculares $\Psi_{\mathrm{n}}$. Para cumprir o critério de máxima simetria na 


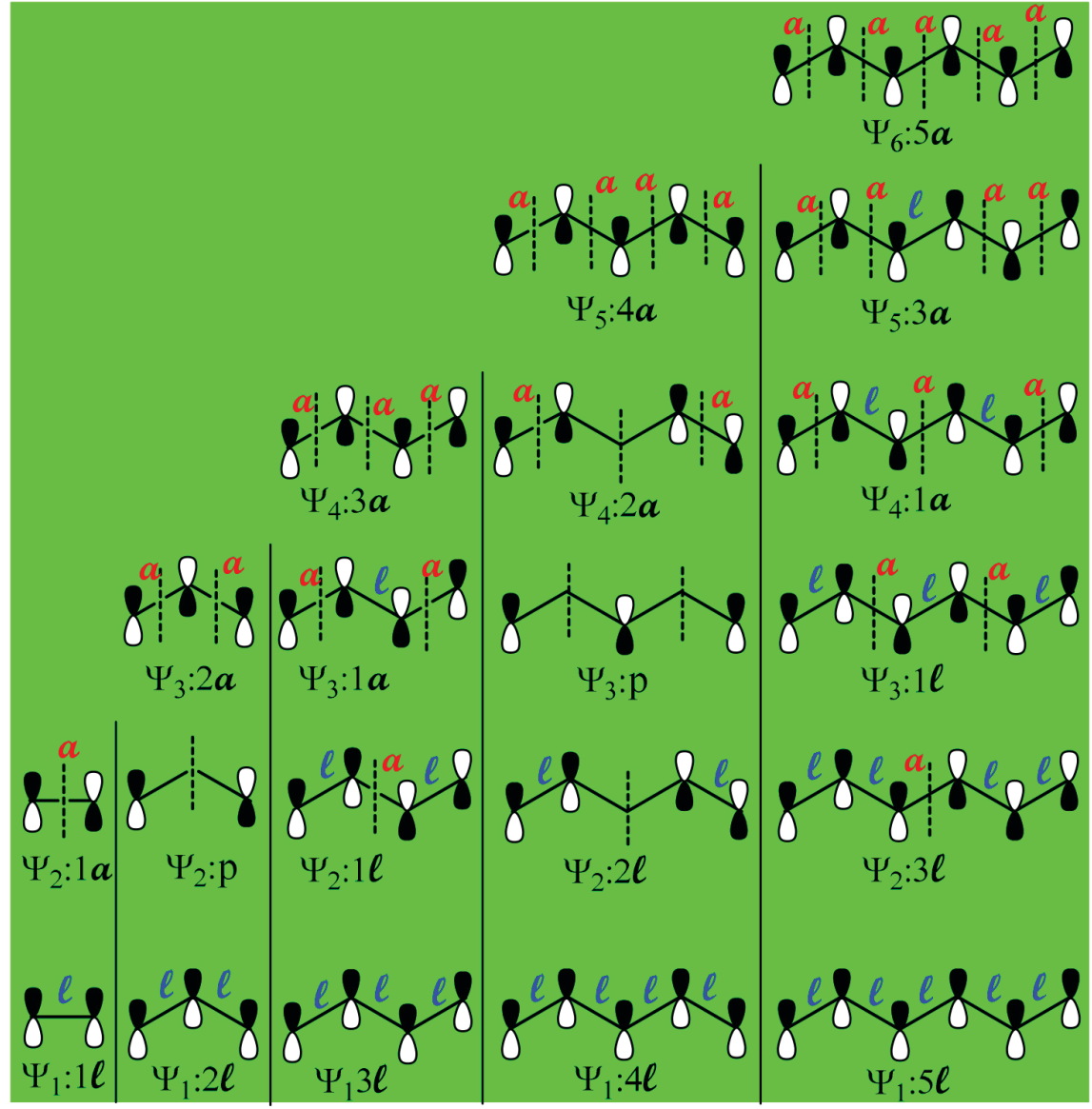

Figura 5. Topologia e energias relativas dos orbitais $\pi$ para moléculas e íons acíclicos conjugados de 2 a 6 átomos empregando o procedimento alternativo
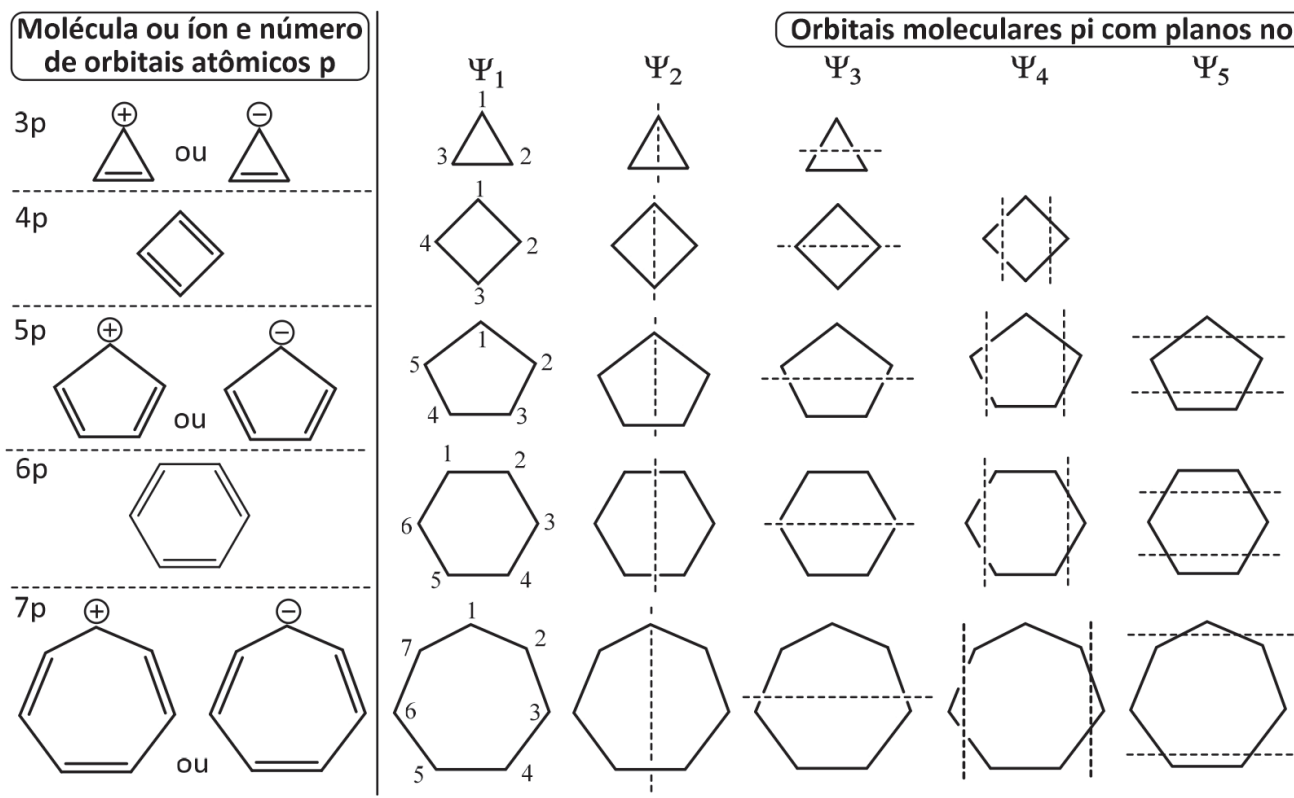

Figura 6. Localização dos planos nodais dos orbitais $\pi$ para moléculas e íons cíclicos conjugados de 3 a 7 átomos empregando o procedimento alternativo dos Passos 1-3

localização de número ímpar de planos nodais, alguns planos podem ficar localizados diretamente sobre um ou dois átomos da estrutura em alguns $\Psi_{\mathrm{n}}$ ao passar por seu centro geométrico, dividindo-a ao meio.

O recomendável é que o estudante inicie a análise pelo anel de três átomos, aproprie-se do procedimento descrito nos Passo 1-3 da Figura 3 e então aplique-os ao anel imediatamente superior em número de átomos, fazendo toda a análise até o anel de sete átomos, um de cada vez. O resultado apresentado na Figura 7 deve ser reproduzido antes que o estudante avance na dedução das energias de cada orbital, para que possa seguir com segurança na etapa da comparação das energias do sistema cíclico com o acíclico abaixo descrita. 
De posse das topologias e deduzidas as energias dos orbitais moleculares dos sistemas acíclicos e cíclicos, é possível proceder à comparação da estabilidade relativa destas duas classes de polienos conjugados.

\section{Comparação das energias relativas dos orbitais de polienos e íons cíclicos e acíclicos empregando a estratégia alternativa e consequências químicas}

Uma vez deduzida a topologia dos orbitais moleculares dos polienos, é possível avaliar a energia relativa dos orbitais moleculares dos orbitais moleculares $\pi$ das substâncias e íons insaturados conjugados cíclicos e acíclicos, através da estratégia alternativa, e agora comparar a estabilidades das mesmas.

O método aqui proposto guarda analogia com os aspectos qualitativos do método de Hückel, ${ }^{16}$ no qual são considerados apenas os elétrons $\pi$ dos sistemas conjugados. Também a quantificação da energia dos orbitais moleculares é feita em unidades intrínsecas do método (unidades de $\alpha$ e $\beta$ no método de Hückel, e unidades de interações ligantes $\boldsymbol{\ell}$ e antiligantes $\boldsymbol{\alpha}$ no método qualitativo alternativo aqui proposto).

Para um dado orbital, quanto maior o número de interações ligantes $\ell$ menor é a sua energia e, ao contrário, quanto maior o número das interações antiligantes $\boldsymbol{a}$ maior é a energia do orbital $\Psi_{n}$, levando-se em conta que uma interação $\ell$ é cancelada por uma $\boldsymbol{a}$. Para uma espécie química específica, quanto maior o número de interações ligantes $\boldsymbol{\ell}$ mais estável ela é.

Como cada elétron assume a energia do orbital que ocupa, na avaliação da energia de cada orbital é levado em consideração a quantidade dos elétrons e a correspondente energia de cada orbital para o somatório e a designação da energia eletrônica total (EET) dos elétrons $\pi$ de cada molécula ou íon. Dessa forma, a EET é o somatório da energia de cada orbital molecular ocupado multiplicado pelo número de elétrons destes orbitais.

Na Figura 8 é apresentado o diagrama de energia dos orbitais dos alcenos conjugados de quatro a oito átomos de carbono, e do eteno para fins de comparação, cujos valores de energia de cada orbital molecular foram deduzidos de acordo com a Figura 5. Para um dado orbital ocupado $\Psi_{n}$ de energia maior, há a repulsão com os elétrons do orbital molecular ligante imediatamente inferior $\Psi_{n-1}$. É por este motivo que o orbital $\Psi_{2}$ do butadieno, apesar de ter o valor algébrico $1 \ell$ igual ao orbital $\Psi_{1}$ do eteno, encontra-se em patamar energético superior. É também por este motivo que os orbitais ocupados dos demais alcenos conjugados têm a posição relativa como indicado na Figura 8. Além disto, nos polienos acíclicos conjugados há a simetria na distribuição da energia dos orbitais ligantes e antiligantes em relação ao ponto central do diagrama de energia, que corresponde à energia do orbital atômico $\mathrm{p}$ do carbono. Dessa forma, quanto maior a energia de um dado orbital molecular ligante menor é a energia do seu correspondente antiligante, e fica fácil perceber que quanto maior for a energia do HOMO menor será a energia do LUMO de um dado polieno.

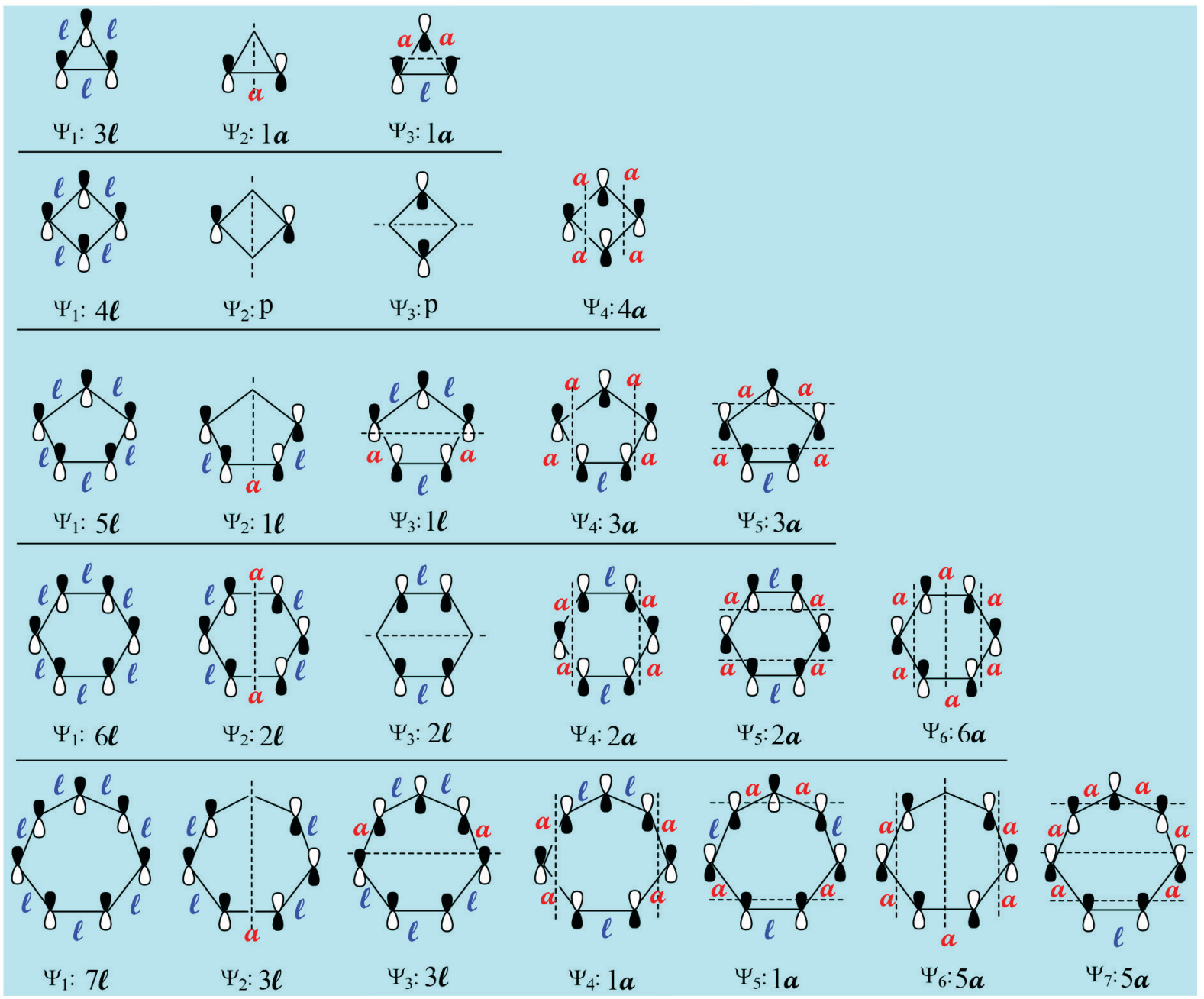

Figura 7. Topologia dos orbitais $\pi$ e dedução das energias relativas para moléculas e íons cíclicos conjugados de 3 a 7 átomos empregando o procedimento alternativo 
Uma consequência do arranjo energético deduzido para os polienos acíclicos conjugados é que o aumento do grau de conjugação faz diminuir a banda de energia HOMO-LUMO, o que justifica o fato de produtos naturais que apresentam esta unidade estrutural absorvam na região do visível, sendo corantes naturais, como o licopeno, o betacaroteno e a bixina, por exemplo. Outra consequência que emana da análise da Figura 8 é a dedução da maior reatividade dos polienos de maior grau de conjugação nas reações onde se comportam como nucleófilos, pois maior é a energia do HOMO em função do incremento do grau de instauração, sendo assim mais fácil doar os elétrons mais externos.

Um aspecto que é ensinado nos cursos de química orgânica é que o aumento da conjugação estabiliza melhor o conjunto dos elétrons $\pi .^{10}$ Para explicar este fato, os textos de graduação lançam mão da teoria de ressonância, justificando que quanto maior o número de estruturas de ressonância para um polieno conjugado mais estável ele é, e assim discrimina-se a maior estabilidade do polieno conjugado em relação ao não conjugado. Propomos aqui empregar o método alternativo para ampliar a explicação através dos orbitais moleculares. Para isto é necessário comparar as energias deduzidas na Figura 8. Entretanto, a comparação direta da EET entre os polienos acíclicos conjugados diferentes não pode ser feita, pois tanto a quantidade de elétrons quanto de carbonos varia ao longo da série. É necessário normalizar o valor da EET por cada átomo de carbono do sistema conjugado. Empregando os dados da Figura 8 e exemplificando com o 1,3-butadieno e o 1,3,5-hexatrieno, a razão EET/ $\mathbf{n}^{\circ}{ }_{\text {Carbono }}$ para o 1,3-butadieno e o 1,3,5-hexatrieno é dada por $8 \ell / 4$ e $18 \ell / 3$, o que corresponde à razão de $2 \ell / C$ e $3 \ell / C$, respectivamente. Assim, fica explicitado que o hexatrieno estabiliza mais o conjunto de elétrons $\pi$ que o butadieno, por unidade de átomo de carbono do sistema conjugado. Aplicando esta linha de raciocínio para polienos acíclicos conjugados superiores, chega-se à conclusão de que o aumento da conjugação estabiliza melhor o conjunto dos elétrons $\pi$.

Uma vez compreendido o diagrama de energia para os polienos conjugados acíclicos da Figura 8, é possível empregar o mesmo método na construção do diagrama dos polienos cíclicos, levando-se em consideração o valor da energia de cada orbital deduzido de acordo com a Figura 7. Para fins de exemplificação, na Figura 9 são apresentados, num mesmo diagrama de energia, os orbitais moleculares

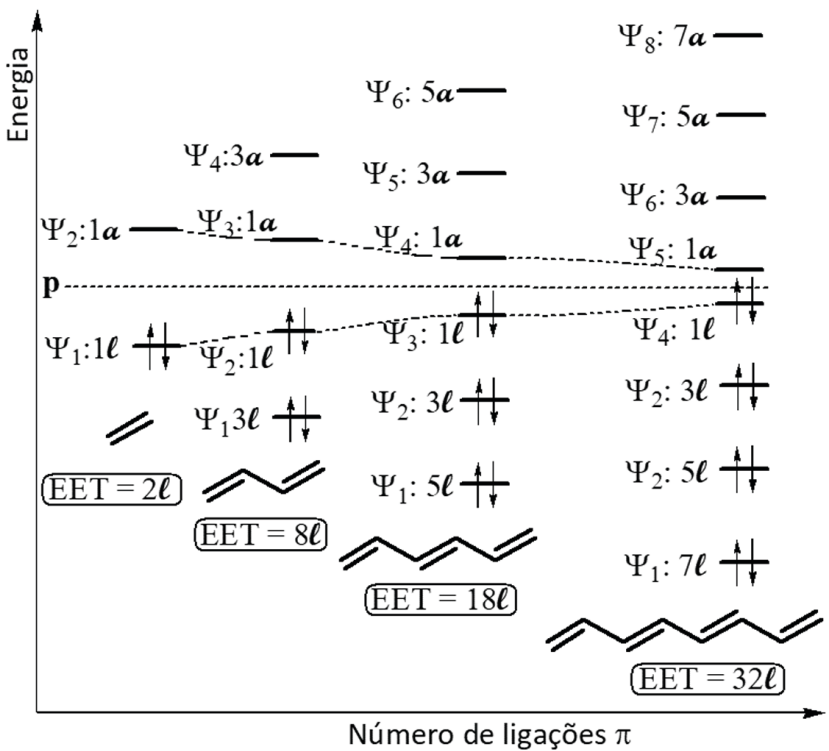

Figura 8. Comparação da energia relativa dos orbitais $\pi$ e da energia eletrônica total (EET) de alcenos empregando o procedimento alternativo

do 1,3,5-hexatrieno e do benzeno, e também do par 1,3-butadieno/ ciclobutadieno, para assim explicitar as consequências eletrônicas da aromaticidade e da anti-aromaticidade.

No cômputo da EET do benzeno, os seis de átomos de carbono são capazes de estabilizar mais intensamente a mesma quantidade de elétrons $\pi$ que o mesmo número de átomos no 1,3,5-hexatrieno, uma vez que a EET destas duas moléculas são $20 \ell$ e $18 \boldsymbol{\ell}$, respectivamente, Figura 9. Como o número de carbonos é o mesmo, podemos aplicar diretamente estes valores como indicativos da maior estabilidade do benzeno em relação ao polieno acíclico; ao se aplicar a razão EET $/ \mathrm{n}^{\circ}{ }_{\text {Carbono }}$, chega-se ao valor $3,3 \boldsymbol{\ell}$, que é $10 \%$ superior ao do 1,3,5-hexatrieno. Por ambas as análises se conclui que essa melhor acomodação dos elétrons $\pi$ no benzeno, consequência da existência de orbitais degenerados e da menor energia relativa destes elétrons, é função da sua aromaticidade.
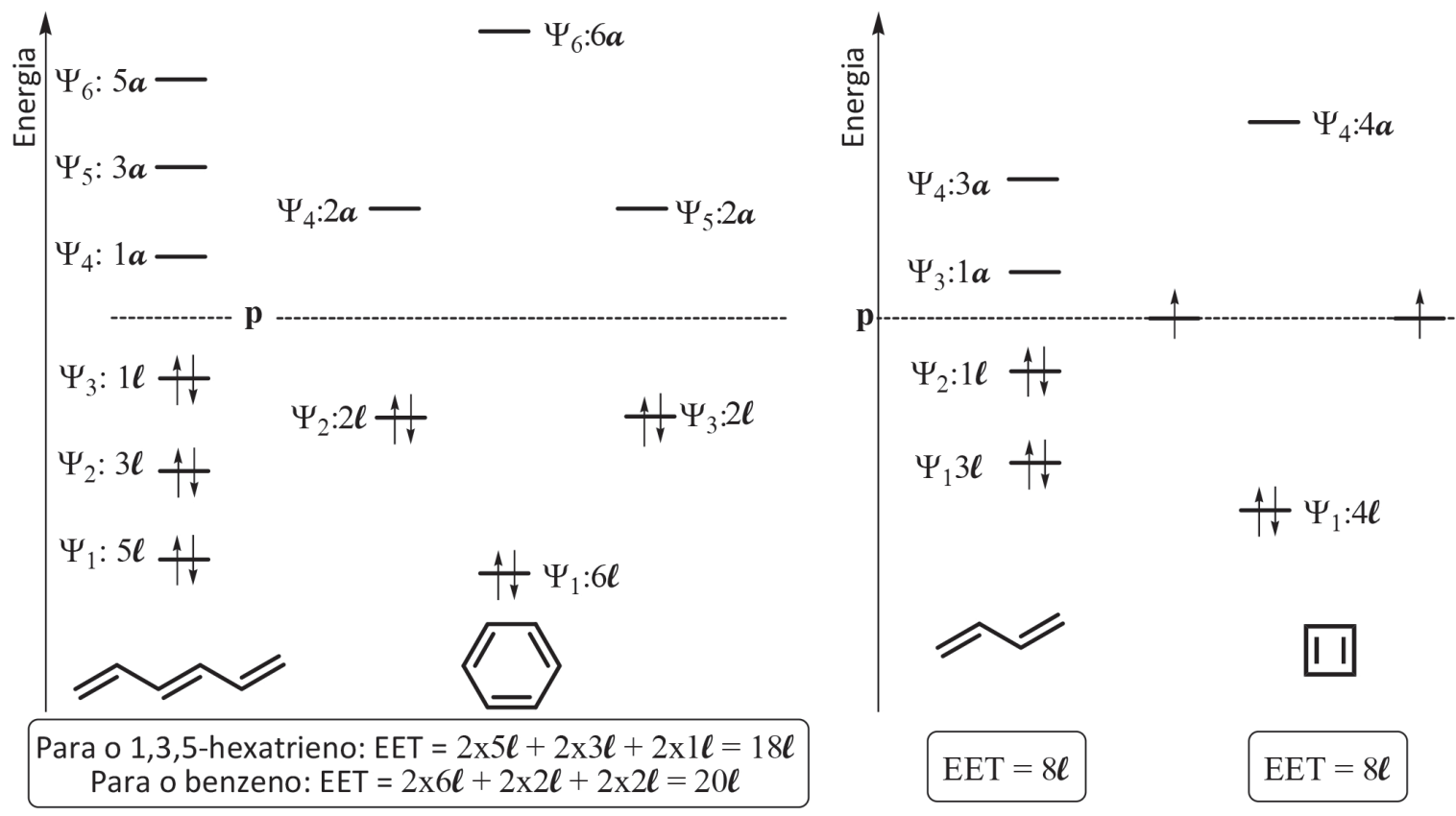

Figura 9. Comparação das energias relativas dos orbitais $\pi$ e da energia eletrônica total (EET) entre benzeno/1,3,5-hexatrieno e entre ciclobutadieno/1,3-butadieno empregando o procedimento alternativo 
Para ilustrar a aplicação do método alternativo na análise de antiaromaticidade, é feita a comparação do ciclobutadieno com o 1,3-butadieno na Figura 9. Pelo método alternativo chega-se ao mesmo valor de EET para as duas substâncias e não num valor menor para o polieno cíclico, como evidenciado para o par benzeno/1,3,5-hexatrieno. $\mathrm{O}$ fato do ciclobutadieno não estabilizar os elétrons $\pi$ mais intensamente que o correspondente polieno acíclico é agravado pela verificação da existência de dois orbitais moleculares degenerados cujas energias são de igual valor ao do orbital atômico p do carbono, e que dois elétrons desemparelhados ocupam estes dois orbitais moleculares, o que confere um caráter de biradical à substância. Todos estes aspectos explicam o comportamento antiaromático do ciclobutadieno e, portanto, sua maior instabilidade em comparação ao 1,3-butadieno, que possui todos os elétrons $\pi$ emparelhados e num patamar energético inferior ao do orbital atômico p do carbono.

\section{CONCLUSÃO}

A abordagem didática desenvolvida conduz o estudante à autonomia na análise da aromaticidade e suas consequências, pois permite que deduza a topologia dos orbitais $\pi$ de moléculas, cátions e ânions de polienos acíclicos e cíclicos e, em seguida, deduza as energias relativas dos orbitais $\pi$ destes sistemas conjugados, conclua por ocorrência de degenerescência como no benzeno e análogos, e assim possa comparar a estabilidade relativa de espécies químicas e julgar aromaticidade e antiaromaticidade. O estudante pode aplicá-la e não ser refém das apresentações estáticas dos textos de graduação (e muitos de pós-graduação) que apresentam estes fatos como coisa pronta.

\section{AGRADECIMENTOS}

O autor agradece o suporte financeiro do Conselho Nacional de Desenvolvimento Científico e Tecnológico - CNPq, da Coordenação de Aperfeiçoamento de Pessoal de Nível Superior - CAPES, e da Fundação de Amparo à Pesquisa do Estado da Bahia - FAPESB. Também agradecemos ao CNPq a bolsa de produtividade em pesquisa e ao Instituto Nacional de Ciência e Tecnologia-INCT em Energia e Ambiente.

\section{REFERÊNCIAS}

1. Halford, B.; Chem. Eng. News 2016, March 28, 24. Para um editorial interessante sobre dificuldade de aprendizado relacionado à existência de conflito de informação química entre livros-texto, e-book e informações da Internet ver: Pienta, N. J.; J. Chem. Educ. 2018, 95, 187.

2. Sendur, G.; Toprak, M.; Chem. Educ. Res. Pract. 2013, 14, 431; Grove, N. P., Bretz. S. L.; Chem. Educ. Res. Pract. 2012, 13, 201.

3. Araujo Neto, W. N.; Quim. Nova Esc. 2007, 13; Pessoa Jr, O.; Quim. Nova Esc. 2007, 25; Gois, J.; Giordan, M.; Quim. Nova Esc. 2007, 34.

4. Mullins, J. J.; J. Chem. Educ. 2008, 85, 83.

5. Caramori, G. F.; Oliveira, K. T.; Quim. Nova 2009, 32, 1871; Glukhovtset, M.; J. Chem. Educ. 1997, 74, 132; Stanger, A.; Chem. Commun. 2009, 1939.

6. Cunha, S.; Quim. Nova 2003, 26, 948.
7. Cunha, S.; Quim. Nova, no prelo, doi: 10.21577/0100-4042.20170197.

8. Para contribuição recente de outros autores ver: Martins, B. S.; Lüdtke, D. S.; Moro, A. V.; Quim. Nova 2017, 40, 342; de Freitas, J. J. R.; Avelino, R. A.; Mata, M. M. S.; Santos, C. S.; Almeida, C. L. A.; Freitas, J. C. R.; Freitas Filho, J. R.; Rev. Virtual Quim. 2017, 9, 1597.

9. Pearson, R. G.; J. Chem. Educ. 1981, 58, 753.

10. Allinger, N. L.; Cava, M. P.; de Jongh, D. C.; Johnson, C. R.; Lebel, N. A.; Stevens, C. L.; Química Orgânica, Guanabara Dois: Rio de Janeiro, 1978; Barbosa, L. C. A.; Introdução à Química Orgânica; Pearson: São Paulo, 2011; Bruice, P. Y.; Química Orgânica, 4ª ed., Prentice Hall: São Paulo, 2006, vols. 1 e 2; Carey, F. A.; Química Orgânica, 7 7a ed., McGraw-Hill-Bookmann: São Paulo, 2011, vols. 1 e 2; Streitwieser, A.; Heathcock, C. H.; Kosover, E. M.; Introduction to Organic Chemistry, $4^{\text {th }}$ ed., Macmillan Publishing Company: New York, 1992; Clayden, J., Greeves, N., Warren, S.; Organic Chemistry, $2^{\text {nd }}$ ed., Oxford University Press: Oxford, 2012; Constantino, M. G.; Química Orgânica Curso Básico Universitário, LTC: Rio de Janeiro, 2008, vols. 1 e 2; Klein, D.; Química Orgânica, 2a ed., LTC: Rio de Janeiro, 2016, vols. 1 e 2; McMurry, J.; Química Orgânica, 3ª ed., Cengage Learning: São Paulo, 2016, vols. 1 e 2; Morrison, R. T.; Boyd, R. N.; Organic Chemistry, $6^{\text {th }}$ ed., Prentice Hall International, Inc.: Hoboken, 1992; Pine, S. H.; Organic Chemistry, $5^{\text {th }}$ ed., McGraw-Hill International Edition: Singapore, 1987; Solomons, T. W. G.; Fryhle, C. B.; Química Orgânica, $10^{\mathrm{a}}$ ed., LTC: Rio de Janeiro, 2012, vols. 1 e 2; Vollard, K. P. C.; Schore, N. E.; Química Orgânica: Estrutura e Função, 6a ed., Bookman: São Paulo, 2013.

11. Pauling, L. Sherman, J.; J. Chem. Phys. 1933, 1, 606.

12. Frost, A. A.; Musulin, B.; J. Chem. Phys. 1953, 21, 572.

13. Berson, J. A.; Angew. Chem., Int. Ed. 1969, 8, 781; Berson, J. A.; Chemical Creativity; Wiley-VCH: Weinheim, 1999; Karachalios, A.; Erich Hückel (1896-1980) From Physics to Quantum Chemistry; Springer: New York, 2010.

14. Doering, W. E.; Detert, F. L.; J. Am. Chem. Soc. 1951, 73, 876; Kikuchi, S.; J. Chem. Educ. 1997, 74, 194; Balaban, A. T.; Schleyer, P. v. R.; Rzepa, H. S.; Chem. Rev. 2015, 105, 3436.

15. Nardholm, S.; Bäck, A.; Bacskay, G. B.; J. Chem. Educ. 2007, 84, 1201; Taubmann, G.; J. Chem. Educ. 1992, 69, 96; Vasconcellos, M. L. A. A.; Quim. Nova 2014, 37, 171; Leal, R. C.; Moita Neto, J. M.; Lima, F. C. A.; Feitosa, C. M.; Quim. Nova 2010, 33, 1211; Kutzelnigg, W.; J. Chem. Comp. 2006, 28, 25.

16. Para uma leitura adequada a químicos orgânicos: Gleiter, R.; Haberhauer, G; Aromaticity and Other Conjugation Effects, Wiley-VCH: Weinheim, 2012; Zimmerman, H. E.; Quantum Mechanics for Organic Chemistry, Academic Press: New York, 1975.

17. A topologia deduzida para alguns orbitais moleculares dos polienos cíclicos pelo método alternativo (por exemplo $\Psi_{5}$ do benzeno e $\Psi_{5}$ do íon ciclopentadienila) não é rigorosamente a mesma obtida pelos métodos da química-quântica (ver referências 16 e 10) mas as tendências das energias relativas sim. Como o método proposto permite deduzir qualitativamente a diferença de energia entre polienos acíclicos e cíclicos e leva o estudante a compreender a origem desta diferença, nos parece um preço muito pequeno a pagar pelo caráter simples do método e do benefício pedagógico proporcionado. 